\title{
REGARDS D'ALLEMAGNE \\ LE CINQUANTENAIRE DU DEUXIÈME SEXE
}

Simone de Beauvoir ne fut pas l'objet d'un débat public ni d'un intérêt médiatique prononcés de l'autre côté du Rhin en 1999. Et elle n'est toujours pas connue du grand public ni mentionnée dans les programmes scolaires. ${ }^{1}$ Pourtant, il y eut quelques commémorations et conférences les quelques dernières années, réflétant plus des initiatives privées qu'un phénomène d'intérêt pour Simone de Beauvoir. Et c'est celles-ci que je voudrais évoquer ici - ayant vécu personnellement une trentaine de conférences de début 1999 à 2001 : quels étaient les thèmes débattus, quelles étaient les raisons de ce débat? Peut-on parler de découverte ou redécouverte de Beauvoir ?

Réception du «Deuxième sexe» pendant les «années de plomb» (1949-1960) et dans les années-mouvement (1970-1980) ${ }^{2}$

\footnotetext{
${ }^{1}$ Une petite anecdocte à ce sujet: en 1999, après une matinée Beauvoir que j'avais eue à Düsseldorf, le bureau fiscal s'adressait aux organisateurs, rappelant que «l'artiste Simone de Beauvoir était intervenue et que la somme devant être encaissée par l'artiste était imposable...»!!!

${ }^{2}$ Voir pour l'Allemagne fédérale: Kristina Schulz, Vom Buch zur «Bibel» der Frauenbewegung: «Das andere Geschlecht » in der Bundesrepublik Deutschland, in: Frankreichjahrbuch 1999.

Contribution lors du colloque international: Cinquantenaire du „Deuxième sexe», Paris janvier 1999 ; Unausgeschöpfte Potentiale feministischer Theoriebildung. Ein Gespräch mit Brigitte Weisshaupt in:Die Philosophin, Forum für Theorie und Philosophie, Oktober 1999/ 20. En projet: une anthologie des compte-rendus sur le Deuxième sexe de 1949-1950, par Ingrid Galster.

Voir pour la R.D.A.: Irene Selle, Zur DDR-Rezeption von Das andere Geschlecht. Ein Erfahrungsbericht, in: Die Philosophin, Forum für Theorie und Philosophie, Oktober 1999/20; Irene Selle, Quelques souvenirs personnels concernant la publication et la réception du Deuxième sexe en République démocratique allemande, Colloque international Cinquantenaire du «Deuxième sexe», Paris 1999; Irene Selle, Publication et réception du Deuxième Sexe en R.D.A. - Compte rendu d'une expérience vécue, in: lendemains, Cinquante ans après le Deuxième Sexe : Beauvoir en débats, Stauffenburg Verlag 24. Jg. $1999 / 94$.
} 
Lors de la parution du Deuxième sexe en France (1949), il n'y a pratiquement pas de réactions en Allemagne. Ce n'est qu'après la traduction allemande parue en 1951 que le livre est pris en compte, de manière relativement marginale d'ailleurs, puisque de 1951 à 1956 le tirage n'atteindra pas plus de 14.000 exemplaires. A partir de 1960, le livre existe aussi dans une version abrégée (157 pages), en édition poche. Il faut noter une traduction parfois erronée, interprétant Simone de Beauvoir dans un certain sens. Ainsi, la version allemande ne reprend pas le titre français du Deuxième sexe mais L'autre sexe. Alors que, comme on le sait, le titre avait fait l'objet d'un débat entre Bost, Sartre et Beauvoir et était donc le résultat d'un choix. L'une des phrases-clé: «On ne naît pas femme, on le devient» («Man wird nicht als Frau geboren, man wird es.») fut traduite uniquement dans le sens de l'influence de la société et de la femme victime de l'oppression sociale, ne tenant pas compte de l'idée de responsabilité de la femme elle-même («Man wird nicht als Frau geboren, man wird dazu gemacht.»). De même, le mot homme est parfois faussement traduit dans l'acceptation de mâle (Mann) et non d'être humain (Mensch).

Dans l'Allemagne fédérale d'après-guerre, chrétienne et très patriarcale, la critique vient surtout du côté des Eglises qui reprochent à Simone de Beauvoir son athéisme et sa mise en cause de l'ordre «naturel» des choses, des valeurs de féminité et maternité.

De 1949 à 1968, il n'y a pratiquement pas de débat, même si de nombreuses femmes affirment aujourd'hui avoir lu Le Deuxième sexe - «une lecture privée».

Les idées du Deuxième sexe ne sont pas à l'origine du mouvement féministe. Lié dans sa genèse au mouvement des étudiants socialistes allemands de l'Ouest (SDS), le mouvement féministe allemand n'a dans sa diversité pas revendiqué le féminisme égalitaire de Simone de Beauvoir ni perçu le Deuxième sexe comme une bible du féminisme. Il ne semble pas y avoir eu une influence immédiate, même si le Deuxième sexe fut lu et discuté dans les groupes féministes d'orientation marxiste, à côté de Bebel, Marx, Engels et Clara Zetkin (ainsi à Berlin et à Bonn) C'est seulement dans les années 70, par le biais d'une action et par l'intermédiaire de la journaliste féministe Alice Schwarzer qu'on commença à parler de Beauvoir. L'un des points forts du mouvement féministe allemand fut en 1971 l'action de 371 personnalités avouant avoir avorté (Selbstbezichtigungsaktion: «ich habe abgetrieben»). A l'origine de cette action, «Le Manifeste des salopes», des 343 femmes en France s'accusant d'avortement, soutenu 
par Simone de Beauvoir. Alice Schwarzer, qui avait participé aux actions du mouvement féministe français, reprit alors l'idée du Manifeste pour le transposer en Allemagne, sans évoquer Beauvoir toutefois.

C'est au début des années 80, avec le foisonnement de la littérature féministe, que Beauvoir deviendra le symbole de la femme émancipée et sera considérée comme une grande féministe. Dans le contexte universitaire, Beauvoir n'est pas évoquée, sinon comme compagne de Sartre. A noter la publication d'une monographie sur Beauvoir en 1978 (rororo Hamburg), des entretiens d'Alice Schwarzer avec Beauvoir en 1983, d'une documentation en 1989 (elefanten press Berlin), et de la traduction de la biographie de Claude Francis et Fernande Gontier en 1989 (rororo Hamburg).

En République démocratique allemande, ce n'est que fin 1989, un mois après la chute du Mur de Berlin, que le Deuxième sexe sera mis en vente dans les librairies. Auparavant, il n'y avait certes pas eu de censure officielle, et on pouvait trouver le livre en français et en allemand dans les bibliothèques, mais on ne pouvait l'acheter. L'absence du Deuxième sexe de Beauvoir dans le débat public, dont certaines œuvres autobiographiques et de fiction (critiquant la société capitaliste) sont appréciées, s'explique par la condamnation de l'existentialisme en tant que philosophie bourgeoise et individualiste par Georg Lukacz en 1951, ainsi que par la critique acerbe du parti communiste français reprise par le parti socialiste unifié SED: il s'agirait d'un livre sur le destin des femmes bourgeoises, les prolétaires n'y seraient pas évoquées. Après 1977, ainsi Irene Selle, «à l'exception d'un récit publié en 1983 dans le cadre d'une anthologie, il n'y eut plus aucune œuvre publiée. Les raisons de ce silence étaient surtout liées aux critiques exprimées par Simone de Beauvoir et Jean-Paul Sartre sur la politique de certains Etats socialistes, et à leur sympathie, après 1968, pour l'extrême-gauche et le féminisme.»

A partir du milieu des années 80 , c'est surtout dans les groupes de femmes protestantes que l'on discute le Deuxième sexe. Irene Selle, spécialiste de Beauvoir et qui avait fait plusieurs expertises en faveur de la publication du Deuxième sexe, responsable de la révision de la traduction du Deuxième sexe en 1992, rapporte l'influence limitée de Beauvoir: «Au sein de groupes informels comme celui de Dresde, la prise de conscience du caractère collectif du malaise des femmes eut pour effet de réduire leurs angoisses et de les rendre solidaires.» A partir de 1985, une nouvelle politique culturelle en 
R.D.A. plus ouverte, et influencée par la Pérestroika, devait favoriser la décision pour une publication jusqu'alors repoussée.

La mort de Beauvoir en 1986, médiatisée, a été l'occasion de revenir sur ses œuvres. Euvres qui continuaient de choquer dans les milieux chrétiens, ainsi que le rapporte Rita Süssmuth, ancienne ministre de la Jeunesse, de la Famille, des Femmes et de la Santé de 1985 à 1988, députée du parti chrétien-démocrate CDU au Bundestag. $\mathrm{Si}$ elle ne se rappelle pas avoir entendu un mot de critique alors qu'elle avait affiché Beauvoir sur le mur de son bureau à l'université de Dortmund, - sa rencontre avec Beauvoir datant d'entre 1975 et 1980 l'avait fascinée par la radicalité du projet - elle raconte que ce fut «un désastre» lorsqu'elle déclara à la télévision «avec une naiveté, je l'avoue ajourd'hui, qui peut être mortelle», que Simone de Beauvoir avait joué un rôle important dans sa vie. «On a exigé tout de suite que je quitte le Ministère de la famille. On a demandé ce que le chancelier avait bien pu imaginer en nommant une telle femme ministre... ces milieux qui protestaient ne devaient pas trop savoir quelles étaient les thèses de Simone de Beauvoir. Ils ne l'avaient sans doute pas lue. Mais ils étaient sûrs d'une chose: c'est une femme dangereuse! Ils avaient dû entendre certaines rumeurs, dont la plus scandaleuse pour eux était probablement la campagne pour la liberté de l'avortement. Ils devaient se dire qu'elle était contre la maternité, qu'elle avait détruit la famille, qu'elle avait proclamé la guerre des sexes. Bref, toutes les horreurs imaginables, on les attribuait à Simone de Beauvoir. Et quelque chose de ce genre ne peut pas être un modèle pour un parti chrétien.» ${ }^{3}$

\section{Réception dans les Années-stagnation (1990-2000):}

De 1990 à aujourd'hui, on observe une stagnation du mouvement féministe, la question de l'égalité des femmes n'est plus à l'ordre du jour, et il n'y a plus de grands débats idéologiques. C'est dans ce cadre qu'il faut situer plusieurs publications.

La nouvelle traduction du Deuxième sexe (1992), la publication en allemand des lettres de Beauvoir à Sartre (1997) puis de celles à Nelson Algren («Eine transatlantische Liebe»,1999) ainsi que des biographies de Deidre Bair (1990), de Toril Moi (1997) suscitent bien sûr des compte-rendus. Le côté privé - relations du couple Sartre-

\footnotetext{
${ }^{3}$ Rita Süssmuth: L'importance de Simone de Beauvoir pour la politique des femmes, in: lendemains, Cinquante ans après Le deuxième sexe: Beauvoir en débats, op.cit.
} 
Beauvoir - y est mis au premier plan, correspondant au nouvel engouement du public pour les autobiographies et les biographies, après l'engouement pour les débats philosophiques dans les années 70 .

Le Cinquantenaire du Deuxième sexe est perçu d'abord à travers la France et le colloque de Paris, entraînant quelques émissions à la radio, la publication de plusieurs articles, revues et livres et l'organisation de trois colloques et soirées.

D'après les chiffres donnés par Rowohlt, le tirage du Deuxième Sexe, de la première édition en 1952 à juillet 1999, a atteint 610.000 exemplaires y compris la version abrégée de 1960 (de 1992 à 1998 ce sont 50.000 exemplaires vendus de la nouvelle traduction). Chiffre élevé, mais si l'on compare aux ouvrages de l'écrivaine féministe Benoîte Groult dans les dernières années (des millions d'exemplaires), c'est relativement peu en l'espace d'une cinquantaine d'années. Selon une enquête de Brigitte (2/2000), l'une des plus importantes revues féminines, auprès de ses lectrices, sur la femme symbolisant le Vingtième siècle, Beauvoir obtenait la place numéro 5, après Sophie Scholl, Rosa Luxemburg, Marie Curie, Marion Gräfin Dönhoff, et avant Madonna, Virginia Woolf, Marlene Dietrich, Coco Chanel et Madeleine Albright. Place honorable, puisque trois allemandes sont en tête de liste et que Beauvoir vient directement après Marie Curie. Résultat qui, si l'enquête avait été faite quelques années auparavant, aurait été probablement sensiblement différent.

Plusieurs livres paraissent donc, regroupant les actes de colloques ou analysant les relations du couple Sartre-Beauvoir. ${ }^{4}$ Quelques revues consacrent un dossier au cinquantenaire. ${ }^{5}$

\footnotetext{
${ }^{4}$ Livres:Alice Schwarzer (Hrsg.), Man wird nicht als Frau geboren. 50 Jahre nach dem „Anderen geschlecht“ ziehen Schriftstellerinnen und Politikerinnen gemeinsam Bilanz: Wo stehen die Frauen heute?, Kiepenheuer \& Witsch, Köln 2000; -Walter von Rossum, Simone de Beauvoir und Jean-Paul Sartre, Berlin 1998; Das Paris der Simone de Beauvoir, von Inga Westerteicher, mit einem Vorwort von Florence Hervé, edition ebersbach Dortmund 1999.

${ }^{5}$ Revues avec un dossier Beauvoir:
}

-Die Philosophin, Forum für Theorie und Philosophie, Simone de Beauvoir 50 Jahre Das andere Geschlecht, Oktober 1999/ 20; -lendemains, Cinquante ans après le Deuxième Sexe: Beauvoir en débats, Stauffenburg Verlag 24. Jg. 1999/94

Revues ayant publié des articles sur Simone de Beauvoir: 
Plusieurs conférences et manifestations culturelles marquent l'événement du Cinquantenaire, à l'instar du colloque organisé au mois de janvier 1999 à Paris (celui-ci avait été marqué par une faible participation des Allemandes et deux contributions sur la réception de Beauvoir à l'est et à l'ouest). C'est, en juin 1999, le festival culturel «Hammoniale» de Hamburg où toute une soirée est consacrée à la vie et l'œuvre de Beauvoir, au fameux pacte Sartre-Beauvoir, à «Ecrire et agir», et à l'amour transatlantique Nelson Algren: (Hommage à Simone de Beauvoir. «Der Himmel gehört dem, der fliegen kann»). En octobre 1999, Alice Schwarzer organise à Cologne un colloque avec une vingtaine d'intervenantes de France, des Etats-Unis et d'Afrique sur l'actualité de Beauvoir, les rapports entre sexisme, racisme et antisémitisme, et sur l'influence de Beauvoir sur la politique pratiquée aujourd'hui. Ce colloque a un caractère surtout médiatique. En novembre 1999, Ingrid Galster organisait un Colloque international scientifique à l'Université catholique d'Eichstätt, avec la participation d'une quarantaine de philosophes, d'historiennes, de sociologues, de littéraires en provenance de France, de Suède, d'Angleterre, des EtatsUnis, du Canada et d'Allemagne - parmi elles Elisabeth Badinter, Kate Millett, Michèle Le Doeuff, Margarete Mitscherlich-Nielsen, Margaret Simons et Monique Wittig. Ce colloque, représentant un premier pas «Pour une édition critique du Deuxième sexe», s'était donné une relecture chronologique du Deuxième sexe pour objectif, afin d'éviter les généralités, révélant la complexité de l'œuvre dans le contexte politique et intellectuel de l'époque. ${ }^{6}$

Et puis il faut mentionner les débats organisés par des associations féminines et des centres de femmes ainsi que par des bureaux d'égalité dans les municipalités, par des bibliothèques et les centres d'éducation protestante et de formation continue comme les

-Beiträge zur feministischen Theorie und Praxis, Köln 53/1999. Gisela Medzeg: Glück für das Leben - Freiheit für die Theorie. Simone de Beauvoir und „Das andere Geschlecht“; -Ihrsinn, eine radikalfeministische Lesbenzeitschrift, Nr. 19, Bochum 1999 : Gisela Medzeg, Lesbierinnen und Schülerinnen. Frauenbeziehungen bei Simone de Beauvoir.

-Das Argument Berlin, Nr. 235/2000, Ingrid Galster: Kurz vor dem Brechreiz. Die Rezeption von Beauvoirs Anderem Geschlecht 1949. Les revues féministes Emma (bimensuel) et Wir Frauen (trimestriel) publient plus ou moins régulièrement des articles sur Beauvoir. ${ }^{6}$ Les Actes du colloque seront édités aux éditions L'Harmattan dans la collection Bibliothèque du féminisme. 
Volkshochschulen. Pour moi-même, ce furent une trentaine de débats centrés autour du Deuxième sexe, réflétant souvent la méconnaissance de l'œuvre de Beauvoir, et dans l'ensemble un plus grand intérêt pour la vie privée que pour la philosophie de Beauvoir. Dans ces débats, il faut noter l'âge des participantes: la plupart avaient une cinquantaine d'années et avaient lu Beauvoir dans leur jeunesse - ce qui les avait marqué profondément ; les jeunes brillaient plus ou moins par leur absence, Beauvoir ne semblant pas les concerner. A noter aussi les différences de réception est-ouest: une insistance sur le contenu philosophique et le côté émancipateur de l'œuvre à l'est (Beauvoir représente plus ou moins une découverte) et sur les rapports de sexe du couple Beauvoir-Sartre à l'ouest - dûe sans doute au débat à la mode sur les relations privées des artistes et écrivains (toute une littérature s'est développée là-dessus les dernières années).

\section{Pour une nouvelle réflexion et perception critique}

Pour apprécier la réception et non-réception de l'œuvre de

Beauvoir ces dernières années, il faut replacer celle-ci dans le contexte de la situation du féminisme en Allemagne. A partir de fin 80, les années-phare du mouvement féministe et de la littérature féministe sont bien finies. Le backlash - le retour du bâton - se fait ressentir, le féminisme a perdu de son intérêt. Il y a une saturation des débats idéologiques. On observe une dispersion du mouvement féministe en une multitude de petits projets de femmes plus ou moins subventionnés par l'Etat et une orientation vers les questions concrètes ainsi qu'une institutionnalisation du mouvement féministe, caractérisée entre autre par l'existence de plus de 1.500 bureaux de l'égalité des femmes dans villes et municipalités.

A l'université, Beauvoir a laissé peu de traces et n'est que rarement citée; elle est considérée plutôt comme dépassée, on se penche plus sur le soi-disant french feminism, le féminisme post-moderne de la différence des sexes, estimé plus moderne, de Julia Kristeva, Luce Irigaray et Hélène Cixous.

L'un des problèmes de la quasi non-réception de l'œuvre de Beauvoir semble lié à la présentation de cette œuvre-même présentation ahistorique qui ne peut qu'ennuyer et paraître dépassée, celle-ci ne tenant pas compte de l'époque de la parution du Deuxième sexe et de son caractère novateur, voire révolutionnaire à la fin de la deuxième guerre mondiale. 
Pourtant, il y a lieu de constater dans des cercles - souvent féministes - un nouvel intérêt pour Beauvoir et l'existentialisme. Une certaine nostalgie joue sans doute un rôle, mais surtout la recherche d'une alternative dans le cadre du non-débat philosophique. Les notions d'individualisme et de responsabilité séduisent, répondant à la critique d'une société jugée égoiste. Devant la difficulté de concilier harmonieusement la vie de couple et de travail dans une société de précarité et de mobilité, une recherche d'autres modèles a lieu Beauvoir représentant un autre modèle de vie, une possibilité de vie commune et indépendante tout à la fois.

Replacé dans son contexte social et historique, le Deuxième sexe pourrait avoir un nouvel impact et un avenir. Dans ce cadre, le projet de l'universitaire Ingrid Galster représenterait un nouveau défi: la fondation d'un institut de recherche sur Simone de Beauvoir et le féminisme égalitaire inspiré par elle, avec l'installation d'une bibliothèque consacrée à Beauvoir et une banque de données commentées et la recherche de la réception du féminisme beauvoirien à l'échelle internationale.

Face à la perte de l'histoire et à une vue isolée et fonctionnelle, il faut assurer la mémoire. Ce n'est ni dans l'hagiographie ni dans la condamnation de Beauvoir que se situe l'avenir du débat, mais dans une réflexion critique.

Florence Hervé 